\title{
Improvement of Integrated Ultrasonic Transducer Sensitivity
}

\author{
JIAN-HUA MO, ANDREW L ROBINSON and FRED L TERRY, JR
}

Soltd-State Electronics Laboratory, Department of Electrical Engineering and Computer Science, Unwersity of Michigan. Ann Arbor. MI 48109-2122 (USA)

DALE W FITTING* and PAUL L CARSON

Radiology Physics and Engineering Division, Department of Radology, Unwersity of Michigan Medical Center, Ann Arbor, MI 48104 (US A)

\begin{abstract}
In this paper, we present a micromachined diaphragm structure for integrated ultrasound transducers This structure greatly reduces the parasitic capacitance between the lower electrode and the conductive $\mathrm{S}$ i substrate in a nonmicromachined structure The micromachining improves both sensitivity and minimum detectable signal It also reduces crosstalk between transducer elements
\end{abstract}

\section{Introduction}

The concept of S1-based integrated ultrasound transducers was first realized with a PVDF-MOSFET (POSFET) structure (Fig 1) by Swartz and Plummer in 1979 [1] The POSFET structure combines a PVDF transducer with a MOSFET input amplifier fabricated with conventional integrated circuit (IC) technology With the help of welldeveloped silicon IC technology in design and fabrication, a large number of small-size transducers can be made on an $S_{1}$ substrate with the potential for further integration of on-chip signalprocessing circuitry Possible applications include medical imaging and non-destructive evaluation since, as recent studies show, large arrays of small-size transducers improve image quality [2]

However, as indicated by Swartz and Plummer, some problems exist in the POSFET structure [1] (1) a large parasitic capacitance between the extended lower electrode and the conductive sillcon substrate, which shunts the input to the preamplifier and therefore causes sensitivity loss,

*D W Fitting is now with the National Institute of Standards and Technology (formerly the National Bureau of Standards), Boulder, CO 80303, U S A and (2) lateral propagation of acoustic waves (possibly through the $\mathrm{S}_{1}$ substrate), which causes acoustic crosstalk between the elements in a transducer array As the size of the transducer is scaled down with integration, any sensitivity loss from already small signals may be costly, and close proximity of neighboring transducer elements in an integrated environment may produce more severe crosstalk Furthermore, the relatively high propagation velocity of acoustic waves in Si may seriously limit the acceptance angle of a transducer array through crosstalk

A POSFET structure based on a dielectric substrate has been suggested [1] to deal with the problem of parasitic capacitance In that version, the problem of lateral propagation through the substrate will still remain To our knowledge, no work has been done to deal with these problems

In this paper, we propose a diaphragm structure for integrated ultrasound transducers based on micromachining of $S i$ This structure greatly reduces the aforementioned parasitic capacitance and reduces the lateral propagation of acoustic waves through $\mathbf{S i}$ substrate As compared to a non-micromachined structure, we see improvement in both sensitivity and minimum detectable signals, we have also found a narrower effective width for each micromachined transducer, which is an indirect indication of reduced acoustic crosstalk

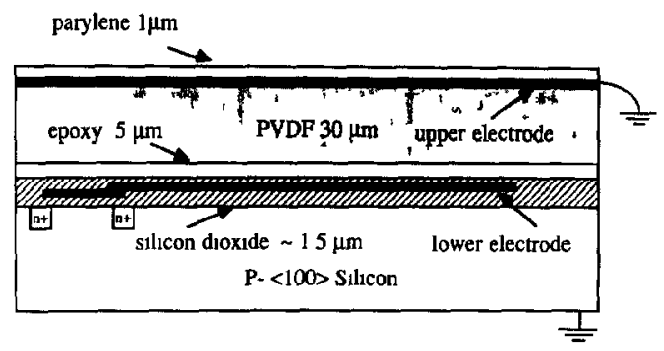

Fig I POSFET structure

(C) Elsevier Sequoia/Printed in The Netherlands 


\section{The Micromachined Diaphragm Structure}

The proposed diaphragm structure is schematically illustrated in Fig 2 This structure is quite similar to the POSFET structure, except that the Si substrate underneath the extended lower electrode and the thin dielectric layer is removed using silicon micromachining technologies [3] and reactive ion etching Because the conductive $S_{1}$ substrate is physically removed, the parasitic capacitance can be basically eliminated except for a component due to peripheral areas For the same reason, the acoustic crosstalk between neighboring transducer elements can also be reduced by the removal of the $S i$ substrate propagation medium for lateral travelling of acoustic waves Therefore the micromachined structure can provide an improvement in transducer sensitivity and a reduction in crosstalk between elements in a transducer array With improved transducer sensitıvity, smaller minımum detectable sıgnals may also be expected

Three sets of transducers have been fabricated (Fig 3) type I-devices with solid substrate and with $\mathrm{S}_{1} \mathrm{O}_{2}$ separating the lower electrode from the Si substrate, type II-devices with a micromachined substrate and with a 1- $\mu$ m-thick stressbalanced composite $\mathrm{SiO}_{2} / \mathrm{Sl}_{3} \mathrm{~N}_{4} / \mathrm{S}_{1} \mathrm{O}_{2}$ diaphragm under the lower electrode, and type III-micromachined devices with the same composite diaphragm and a 5- $\mu \mathrm{m}$-thick $\mathrm{p}^{+}$silicon layer Type III devices are intermediate products in the fabrication of type II devices and are included here to help distinguish the electrical effects of micromachining from the acoustical effects

The fabrication of type I devices starts from a lightly doped p-type $\langle 100\rangle$ silicon substrate A wet oxidation is performed at $1100^{\circ} \mathrm{C}$ for three hours to grow a $\mathrm{SiO}_{2}$ layer of $1 \mu \mathrm{m}$ thick $\mathrm{Cr}$ and $\mathrm{Au}$ are then evaporated on the $\mathrm{SiO}_{2}$ with thicknesses of $400 \AA$ and $2000 \AA$, respectively, and are patterned into $1 \mathrm{~mm}$ squares with extended bonding pads to serve as lower electrodes Next, a $40-\mu \mathrm{m}-$ thick PVDF sheet, with Au on its top as the upper electrode, is bonded onto the $S_{1}$ substrate,

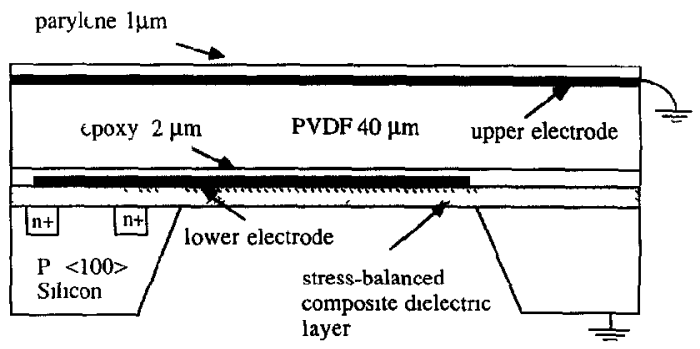

Fig 2 Micromachined diaphragm structure

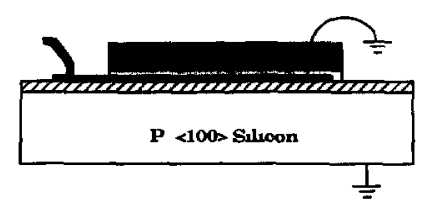

(a)

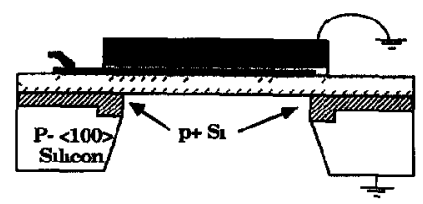

(b)

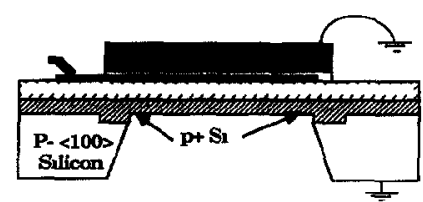

(c)

Fig 3 Three types of transducers with (a) type I-solid substrate, (b) type II-diaphragm w/o5/micron $\mathrm{p}^{+} \mathrm{S}_{1}$ layer, and (c) type III-diaphragm with $5 /$ micron $\mathrm{p}^{+} \mathrm{S}_{1}$ layer

using non-conductive epoxy The upper electrode and the $S_{1}$ substrate are both grounded, and the signal comes out from the lower electrode

There are several different processing steps in the fabrication of types II and III devices as compared to the fabrication sequence of type I devices First, before the formation of the thin dielectric layer, deep and shallow boron diffusions are performed to form a $\mathrm{p}^{+}$rim and a $\mathrm{p}^{+}$silicon layer as mechanical support for diaphragms formed later [4] These diffusions are formed with solid boron sources at $1175^{\circ} \mathrm{C}$ for 35 hours and 16 hours, respectively Second, instead of a single $\mathrm{SiO}_{2}$ layer in type I devices, a short wet oxıdation at $1100^{\circ} \mathrm{C}$ is conducted to form a $2000 \AA \mathrm{S}_{1} \mathrm{O}_{2}$ layer, and then $\mathrm{CVD} \mathrm{Sl}_{3} \mathrm{~N}_{4}$ and $\mathrm{SiO}_{2}$ layers with thicknesses of $1500 \AA$ and $6500 \AA$ are deposited at $820^{\circ} \mathrm{C}$ and $920^{\circ} \mathrm{C}$, respectively These conditions provide a flat composite dielectric layer Third, before bonding the PVDF sheet onto the chip, the Si-etch window on the backside of the wafer is defined with the lower electrode at its center The S1 substrate in the backside etch window is anisotropically etched with a water and EDP solution at $110^{\circ} \mathrm{C}$ The Si-etch will stop at $\mathrm{p}^{+} \mathrm{rim}$ and $\mathrm{p}^{+}$layer with etch stop depth at about $10 \mu \mathrm{m}$ and $5 \mu \mathrm{m}$ respectively, from the front side For type II devices, an additional step is taken after bonding the PVDF sheet, reactive ion etching (RIE) is performed to remove the $5-\mu \mathrm{m} \mathrm{p}^{+}$sll1con layer underneath the lower electrode 


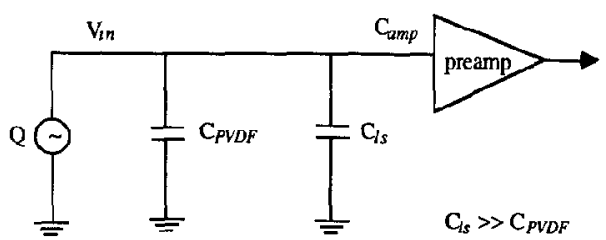

Fig 4 Simplified equvalent circuit

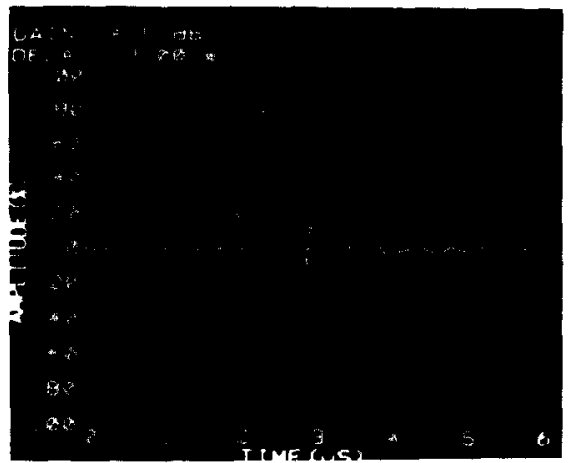

(a)

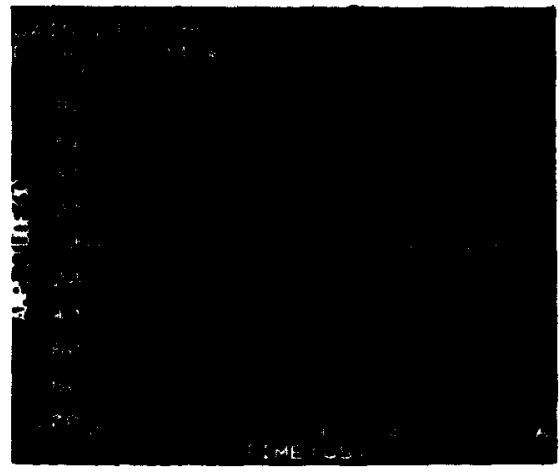

(b)

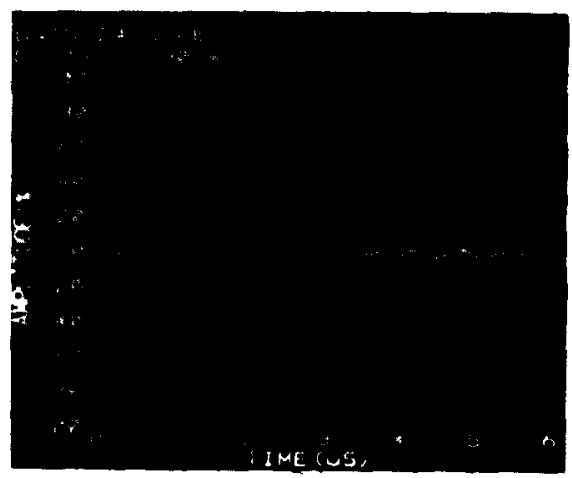

(c)

Fig 5 Typical response waveforms for transducers (a) Type II $D G=145 \mathrm{~dB}, D P=80 \%$, (b) type I $D G=245 \mathrm{~dB}$, $\mathrm{DP}=90 \%$, (c) type III $\mathrm{DG}=245 \mathrm{~dB}, \mathrm{DP}=80 \%$

\section{Simple Modeling and Experimental Results}

\section{Sensitivity and Minumum Detectable Signals}

Measurements of transducer response to acoustical input were performed through a water path on all transducers with an arr backing The device under test drives a simple buffer

Figure 4 shows a simplified equivalent circuit for POSFET structure Based on this simple model, the sensitivity ratio $S R$ for two types of transducers with the same acoustical backing condition is

$S R_{\mathrm{a} / \mathrm{b}} \equiv \frac{S^{\mathrm{a}}}{S^{\mathrm{b}}}=\frac{C_{\mathrm{PVDF}}^{\mathrm{b}}+C_{\mathrm{amp}}^{\mathrm{b}}+C_{\mathrm{ls}}^{\mathrm{b}}}{C_{\mathrm{PVDF}}^{\mathrm{a}}+C_{\mathrm{amp}}^{\mathrm{a}}+C_{\mathrm{ls}}^{\mathrm{a}}}$

where $S^{\mathrm{a}}$ and $S^{\mathrm{b}}$ are the sensitivities of the transducers being compared, $C_{\text {PVDF }}$ is the clamped capacitance associated with PVDF sheet with its area defined by the lower electrode, $C_{\mathrm{ls}}$ is the previously mentioned parasitic capacitance between the lower electrode and the conductive $S_{1}$ substrate, and $C_{\text {amp }}$ is the input capacitance of the pre-amplifier

Figure 5 illustrates typical waveforms for each type of transducer Measured $S R_{\mathrm{II} / \mathrm{III}}=89 \mathrm{~dB}$ and $S R_{1 / / /}=96 \mathrm{~dB}$, in good agreement with predicted values (Table 1) The minimum detectable signals are about $4000 \mathrm{~Pa}, 1600 \mathrm{~Pa}$, and $7000 \mathrm{~Pa}$ (Fig 6) for types I, II, and III devices, respectively We believe that the minımum detectable signals are mostly limited by the noise level of the test set-up With the same air backing for all transducers, the

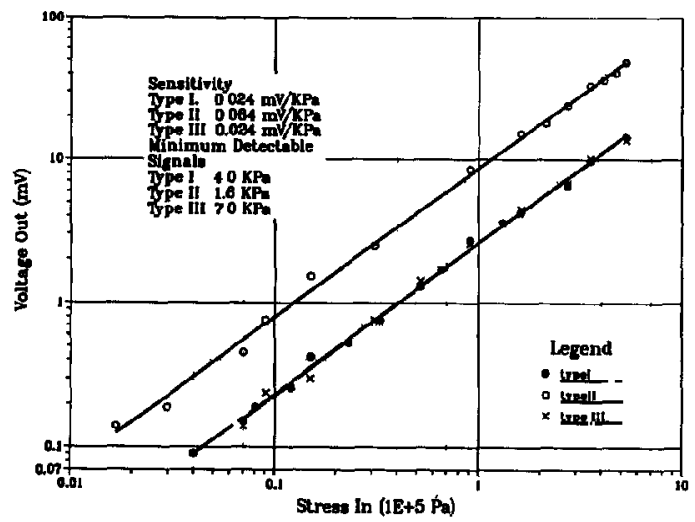

Fig 6 Transducer sensitivity and minmum detectable signals

TABLE 1 Predicted and measured sensitivity ratio (SR) (dB)

\begin{tabular}{lll}
\hline$S R_{\mathrm{a} / \mathrm{b}}$ & Predicated & Actual \\
\hline$S R_{11 / 1}$ & 109 & 89 \\
$S R_{\mathrm{n} / 11 \mathrm{I}}$ & 113 & 96 \\
\hline
\end{tabular}




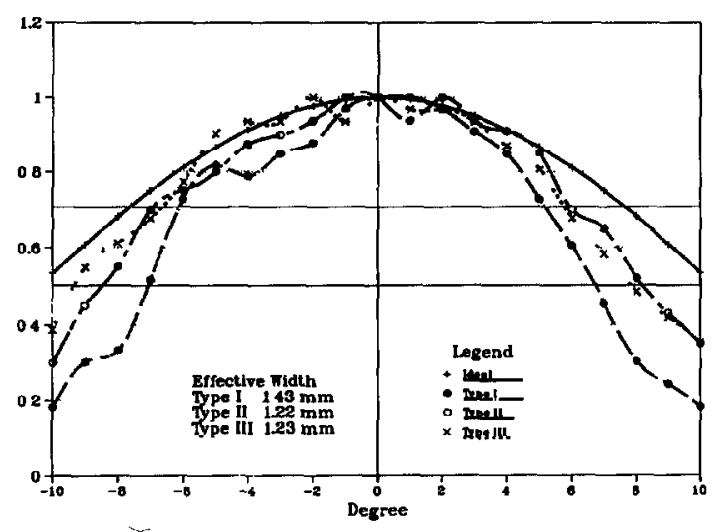

Fig 7 Transducer directiviıy

sensitivity improvement and smaller miminum detectable signal are electrical effe ts of the micromachining of $S_{1}$ substrate rather tha $n$ acoustical in nature

\section{Crosstalk}

In general, crosstalk between transducer elements makes a transducer acoustically wider than it is physically The effective element width can be determined through directivity measurement as an indirect indication of acoustic crosstalk in devices The directivity of each type of transducer was measured by changing angles between the incoming acoustic beam and the transducer under test Figure 7 shows the directivities for the ideal case and for each type of transducers The average of $-3 \mathrm{~dB}$ and $-6 \mathrm{~dB}$ effective widths is about $122 \mathrm{~mm}$ for types II and III devices and $143 \mathrm{~mm}$ for type I devices, evidence of reduced acoustical crosstalk in micromachined transducers

\section{Conclusions}

We have presented a micromachined diaphragm structure for integrated ultrasound transducers This structure greatly reduces a large parasitic capacitance in a non-micromachined structure and thus improves transducer sensitivity and allows a smaller minımum detectable signal It also reduces acoustic crosstalk between transducer elements because the $\mathrm{S}$ i substrate underneath the lower electrode, a lateral acoustic wave propagation medium, is removed The improved device performance through micromachining, coupled with other advances in material quality and process compatibility, may permit development of two-dimensional transducers with better Image quality

The above experimental results are also appl1cable to transducers based on P(VDF-TrFE) copolymer, which is under study The process compatibility provided by $\mathrm{P}(\mathrm{VDF}-\mathrm{TrFE})$ copolymer makes it an attractive alternative to PVDF in terms of further integration of ultrasound transducer with the signal-processing circuitry on the $S_{1}$ wafer [5]

\section{Acknowledgements}

This work was supported in part by the National Science Foundation under contract \# ECS-8616329, by a Biomedical Research Support Grant administrated by the University of Michigan's Vice-President for Research, and by PHS Grant 5R01 HDI7243, National Institute for Human Growth and Development, DHHS

The authors thank the technical staff of the Solid State Electronics Laboratory and Radiology Physics and Engineering Laboratory at the University of Michigan for their assistance in processing and testing $\mathrm{J}-\mathrm{H}$ Mo also acknowledges the support of a fellowship from the Research Partnership Program, sponsored by the University of Michigan Vice-President for Research and the Horace Rackham School for Graduate Studies He would also like to thank Dr J B Fowlkes for numerous helpful discussions

\section{References}

I R G Swartz and J D Plummer, Integrated silicon-PVDF acoustic transducer arrays, IEEE Trans Electron Devices, ED.26 (1979) 1921-1931

2 D W Fitting, P L Carson, J Giesey and P Grounds, A two-dimensional array recelver for reducing refraction artifacts in ultrasome computed tomography of attenuation, IEEE Trans UItrason Ferroelectr Freq Control, UFFC-34 (1987) 346-356

$3 \mathrm{~K} D$ Wise and S K Clark, Diaphragm formation and pressure sensitivity in batch-fabncation silicon pressure sensors, Tech Digest, IEDM, (1978) 96-99

4 I $H$ Chol and $K$ D Wise, A silicon-thermopile-based infrared sensing array for use in automated manufacturing, IEEE Trans Electron Devices, ED-33 (1987) 72-79

5 A Fiorillo, $P$ Dano, J Van der Spiegel, C Domenici and J Foo, Spinned P(VDF-TrFE) copolymer layer for a silıconpiczoelectric integrated US transducer, Proc Ultrasonics Symp, 1987, pp 667-670 